\title{
Enhanced Noise Type Recognition Using Statistical Measures
}

\author{
J Sankara Ganesh ${ }^{1}$ Dr. V. Srinivasa Rao ${ }^{2}$ Dr. K. Srinivas ${ }^{3}$ \\ Post Graduate Student Professor Professor \\ 1, 2, 3 (Department of CSE, VRSEC, Vijayawada, AP, INDIA
}

\begin{abstract}
Noises are the unwanted information in an image, so they should be removed before further processing. Existing methods consider histogram based analysis which is globally varied one. A modified statistical measured based automatic noise type recognition technique is proposed in this paper. This has 2 phases including training phase and testing phase. The key role involves deduction of noise samples using filters like wiener, lee, median and extracts the statistical measures like kurtosis and skewness from samples. Kurtosis and skewness values exhibit behavior based on noise type. By using the statistical information and trained data we can classify the type of noise. Finally the noise type is identified and corresponding filter is applied. Thus noise eliminated image would give the desirable results during further processing. Experimental results show that the method is capable of accurately classifying the types of noise.
\end{abstract}

Keywords: Enhanced Noise Type Recognition, Kurtosis, Noise type identification, Skewness, Statistical features.

\section{Introduction}

Environmental monitoring and earth resource mapping require broad-area imaging at high resolutions. Synthetic Aperture Radar (SAR) acquired imagery in inclement weather or during night as well as day. SAR systems take advantage of long-range propagation characteristics of radar signals and complex information processing capability of modern electronics to provide high resolution imagery. SAR images are used in many fields, most importantly for vegetation mapping, density of the forests and trees outside the forests. Generally the SAR images are comprises of various types of noises and disturbances. Before process the images we have to minimize or reduce the noise density.

Many image de-noising algorithms appeared in the literature for reduction of various types of noises. In general all those algorithms run properly and reduce various types of noises such as Gaussian noise, speckle noise and salt and pepper noise. The underlying algorithms include wiener filter for Gaussian noise, median filter for salt and pepper noise and Lee filter for speckle noise. These techniques are very useful for applications where manual de-noising is acceptable. For this approach we have to identify the type of noise manually and apply the corresponding algorithm [1]. Automatic technique for identification of type of noise is required. Because once the type of noise is identified from the given image an appropriate algorithm is applied. Only few researches addressed this issue to date because of complicated features.

Histogram analysis is also preferred to identify noise type. The detection criterion of impulsive noise includes if $\frac{\operatorname{mean}(D(n))}{\max (\mathbb{D}(n))}>\lambda$ where $\lambda=0.9$. The line passing through $(0,0)$ characterizes the presence of multiplicative noise in image otherwise it indicates Gaussian noise [2]. This method is easy to implement but varied among images. The main drawback of this method is histogram variation. In the case of multiplicative noise in most cases the line is not passing through $(0,0)$ where it violates method.

Noise Models:

Noise is any undesired information that contaminates an image from various sources. The digital image acquisition process, which converts an optical image into a continuous electrical signal that is then sampled, is primary process by which noise appears in digital image [3].

\section{1) Gaussian Noise}

This type of noise is also called the Gaussian noise or normal noise is randomly occurs as white intensity values. Gaussian distribution noise can be expressed by:

The Gaussian distribution in 2-D,

$$
\begin{aligned}
& G(x, y)=\frac{1}{2 \Pi \sigma^{2}} e^{-\frac{x^{2}+y^{2}}{2 \sigma^{2}}} \\
& f(I, j)=y(I, j)+w(I, j) \\
& \text { where } 1 \leq \mathrm{i} \leq \mathrm{M}, 1 \leq \mathrm{j} \leq \mathrm{N}
\end{aligned}
$$
image.

Let $\mathrm{M}$ and $\mathrm{N}$ be the size of the original image $\mathrm{y}(\mathrm{i}, \mathrm{j}), \mathrm{w}(\mathrm{i}, \mathrm{j})$ be the noisy image and $f(i, j)$ is the noisy 


\section{2) Salt \& Pepper Noise}

Occurrences of both black and white intensity values, and caused by threshold of noise image [4]. Salt $\&$ pepper distribution noise can be expressed by:

$$
f(I, j)=y(I, j)+w(I, j)
$$

\section{3) Speckle Noise}

Speckle noise in conventional radar results from random fluctuations in the return signal from an object that is no bigger than a single image-processing element. It increases the mean grey level of a local area. This is due to stripes or missing data [5].

$f(i, j)=y(i, j) * w(i, j)$

Let $y_{i j}$ be the gray level of a true image $y$ at pixel location $(i, j), \omega_{i j}$ be the gray level of the noisy image $\omega$ at pixel $(\mathrm{i}, \mathrm{j}), r_{i j}$ are random numbers and $\mathrm{r}$ is the noise ratio.

\section{Noise Filters:}

1) Wiener Filter: Gaussian noise can be removed using wiener filter. Its approach is minimizing Mean Square Error. The goal of the Wiener filter is to filter out noise that has corrupted a signal. It is based on a statistical approach. Gaussian is smoothing filter in the $2 \mathrm{D}$ convolution operation that is used to remove noise and blur from image. . The Wiener filter is the mean square error-optimal stationary linear filter for images degraded by additive noise and blurring.

2) Lee Filter: Lee filter is convolved within the spatial domain. The Lee filter is an adaptive filter, which changes according to the local statistics of the current pixel. The Lee multiplicative filter is based on a multiplicative noise image model [10].

3) Median Filter: Median filter is the non-linear filter which changes the image intensity mean value if the spatial noise distribution in the image is not symmetrical within the window. Median filter reduce is the variance of the intensities in the image. Median filter is a spatial filtering operation, so it uses a 2-D mask that is applied to each pixel in the input image. To apply the mask means to centre it in a pixel, evaluating the covered pixel brightness and determining which brightness value is the median value [7].

Instead of simply replacing the pixel value with the mean of neighboring pixel values, it replaces it with the median of those values like in equation 4. The median is calculated by first sorting all the pixel values from the surrounding neighborhood into numerical order and then replacing the pixel being considered with the middle pixel value.

$$
y[m, n]=\operatorname{median}\{x[i, j]\},(i, j) \varepsilon w
$$

where $\mathrm{w}=$ window size of $\mathrm{mxn}$ image

1. Proposed Approach - Training Phase

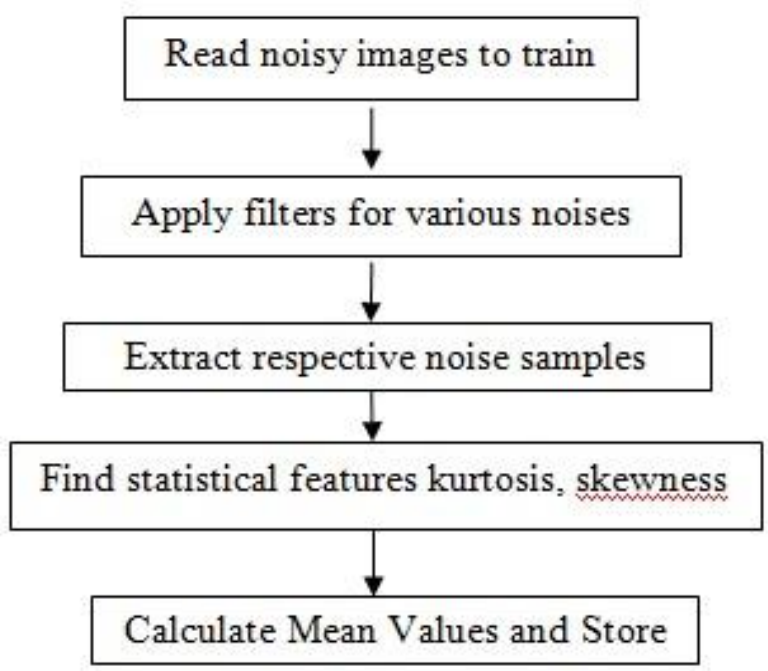

Fig 1: Training Phase

2 Testing Phase 


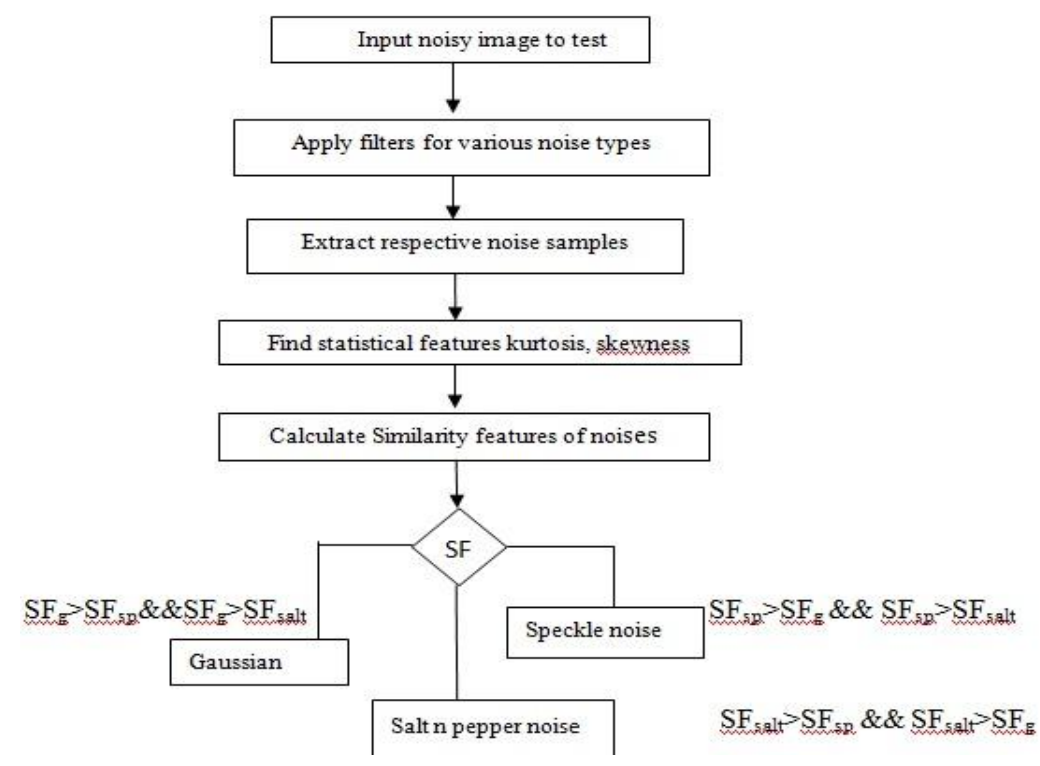

Fig 2: Testing Phase

The procedure can be summarized as follows:

First generate training sequences of various images for each noise type. Consider the original $600 \times 600$ image $y(i, j)$ is contaminated by either Gaussian or speckle type of noise, $\omega(i, j)$. Thus the observed image $f(i, j)$ can be modeled as equation (1) for additive noise, equation (2) for impulsive noise and equation (3) for multiplicative noise. To start with it has been assumed that the type of noise is unknown, but it belongs to one of 3 known classes defined from trained data. For each type of noise, choose a simple linear or nonlinear spatial filter operator capable of removing most of the noise type from the image. Suppose $H_{k}(i, j)$, where $1 \leq k \leq 3$, denote these filter operators. To extract some noise samples, first process the image through each filter operator to obtain. That three selected filters, Wiener filter, Lee filter and Median filter are applied to the noisy image $f(i$, j) to get three different estimates $y_{n}(i j)$, the original image $y(i, j)$ as follows:

$\bar{y}_{\text {wiener }}(\mathrm{i}, \mathrm{j})=\mathrm{f}(\mathrm{i}, \mathrm{j}) * \mathrm{H}_{\text {wiener }}(\mathrm{i}, \mathrm{j})$

$\bar{y}_{\text {lee }}(\mathrm{i}, \mathrm{j})=\operatorname{Exp}\left[\log \left(\mathrm{f}(\mathrm{i}, \mathrm{j}) * \mathrm{y}_{\text {lee }}(\mathrm{i}, \mathrm{j})\right)\right]$

$\bar{y}_{\text {median }}(\mathrm{i}, \mathrm{j})=\mathrm{f}(\mathrm{i}, \mathrm{j}) * \mathrm{H}_{\text {median }}(\mathrm{i}, \mathrm{j})$

In equation (6) logarithm and exponential operations are needed to perform Lee filtering to remove the multiplicative noise. First image is converted into logarithmic value and exponential value is calculated. Then, three noise samples from the three filters are obtained as follows:

$\omega_{\text {wiener }}=\mathrm{f}(\mathrm{i}, \mathrm{j})-\bar{y}_{\text {wiener }}(\mathrm{i}, \mathrm{j})$
$\omega_{\text {lee }}=\mathrm{f}(\mathrm{i}, \mathrm{j}) / \bar{y}_{\text {lee }}(\mathrm{i}, \mathrm{j})$
$\omega_{\text {Medianr }}=\mathrm{f}(\mathrm{i}, \mathrm{j})-\bar{y}_{\text {Median }}(\mathrm{i}, \mathrm{j})$

Gaussian noise and impulsive noise are additive in nature. So to extract noise samples, subtract filter output from original image. As speckle is multiplicative in nature division method is applied. Estimate statistical features kurtosis and skewness of each filtered noise sequence and compute their average to yield the reference values of noise samples. Kurtosis and skewness are main statistical features of image analyzed from image histogram.

\section{Kurtosis}

\section{The Statistical Features}

Kurtosis is any measure of the "peakedness" of the probability distribution of a real-valued random variable. It is a descriptor of the shape of a probability distribution and there are different ways of quantifying it for a theoretical distribution and corresponding ways of estimating it from a sample from a population.

$\operatorname{Kurt}(\mathrm{X})=\mathrm{E}\left[(\mathrm{X}-\mu)^{4}\right] / \sigma^{4}$

\section{Skewness}

Skewness is a measure of the asymmetry of the probability distribution of a real-valued random variable. The skewness value can be positive or negative, or even undefined. Many models assume normal distribution; i.e., data are symmetric about the mean [4]. Skewness of the dataset indicates whether deviations from the mean are going to be positive or negative. 


\section{Evaluation of the features}

The evaluation of features is carried out using Matlab simulations. The functions kurtosis(X) and skewness $(\mathrm{X})$ are used to calculate the respective values. A range of feature values are calculated for each image sample and then the mean value is calculated using the mean $(\mathrm{x} 1, \mathrm{x} 2, \mathrm{x} 3 \ldots)$ function which returns the mean value of input specified. Repeat the procedure for each noise to find mean value of kurtosis and skewness as specified in Fig 1.

Store mean values of kurtosis and skewness of each filter as reference values. The image to be tested is applied same procedure and probabilities are calculated using following formulae. Similarity features using kurtosis and skewness of various filters are calculated.

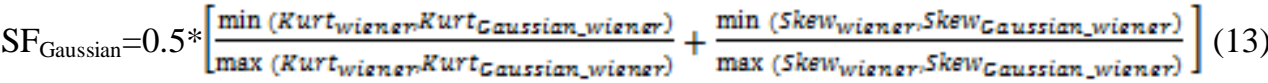

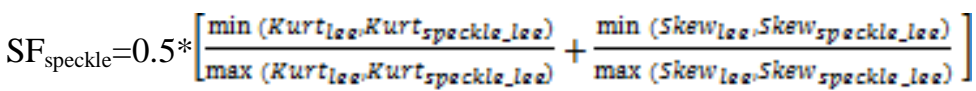

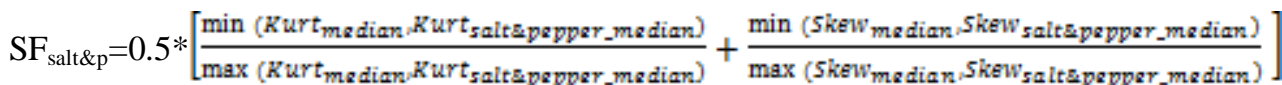

Similarity features indicate $\min / \max$ value of kurtosis and skewness and their average. Generally $\min / \max$ value returns probability of particular feature. As if coin has probability of $1 / 2$ where $\min =1$ and $\max =2$.

if Kurt $_{\text {median }}>\mathrm{T}$

$$
\mathrm{N}_{\text {type }}=\text { Salt } \mathrm{n} \text { pepper Noise }
$$

else if $\mathrm{SF}_{\text {Salt\&pepper }}>\mathrm{SF}_{\text {Gaussian }} \& \& \mathrm{SF}_{\text {Salt\&pepper }}>\mathrm{SF}_{\text {speckle }}$

$\mathrm{N}_{\text {type }}=$ Salt $\mathrm{n}$ pepper noise

else if $\mathrm{SF}_{\text {Gaussian }}>\mathrm{SF}_{\text {speckle }} \& \& \mathrm{SF}_{\text {Gaussian }}>\mathrm{SF}_{\text {salt\&pepper }}$

$\mathrm{N}_{\text {type }}=$ Gaussian Noise

else

$$
\mathrm{N}_{\text {type }}=\text { Speckle Noise }
$$

(In equation (14), Kurt lee $_{\text {and }}$ Skewlee denote Kurtosis and Skewness of $\omega_{\text {lee }}$, Kurt speckle lee and Skew speckle_lee denote the expected Kurtosis and Skewness of $\omega_{\text {Lee }}$ if the original noise $\omega(i, j)$ is actually Speckle noise. Minimum and maximum operations are used to assure that the maximum similarity value is 1 [5]. The similarities of Kurtosis and Skewness are equally weighted to get $\mathrm{SF}_{\text {speckle, the final measure of similarity }}$ between $\omega_{\text {lee }}$ and actual speckle noise. The same procedure is carried out to obtain the expected Kurtosis and Skewness values for Salt \& pepper noise and Gaussian noise.

Compare similarity features to get noise type. If kurtosis value of median filtered output is greater than threshold $\mathrm{T}$ then it is salt $\mathrm{n}$ pepper noise for 600x600 images. Otherwise if similarity features of Gaussian noise is more than that of remaining noises then noise type is Gaussian noise. Same is repeated for Salt \& pepper and speckle noises. But in Simple Pattern Classification Approach [1], based on kurtosis of median filtered output similarity values of Gaussian and Speckle are set to be zero initially which is not appropriate for all images.

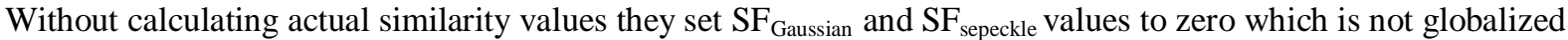
one. But in our proposed method, similarity values of all noises are calculated first and compared later. To identify salt $\mathrm{n}$ pepper noise here we propose two methods. First includes kurtosis value of median filtered output is greater than threshold and second one comparing similarity values. Here threshold $\mathrm{T}$ value is set to be 25 because threshold value depends on size of image. Here we consider noisy images of 600x600 sizes thus setting threshold value 25 . We tested our proposed method for 24 images with success rate $100 \%$. The results for tested images are summarized in TABLE 1. 


\section{RESULTS}

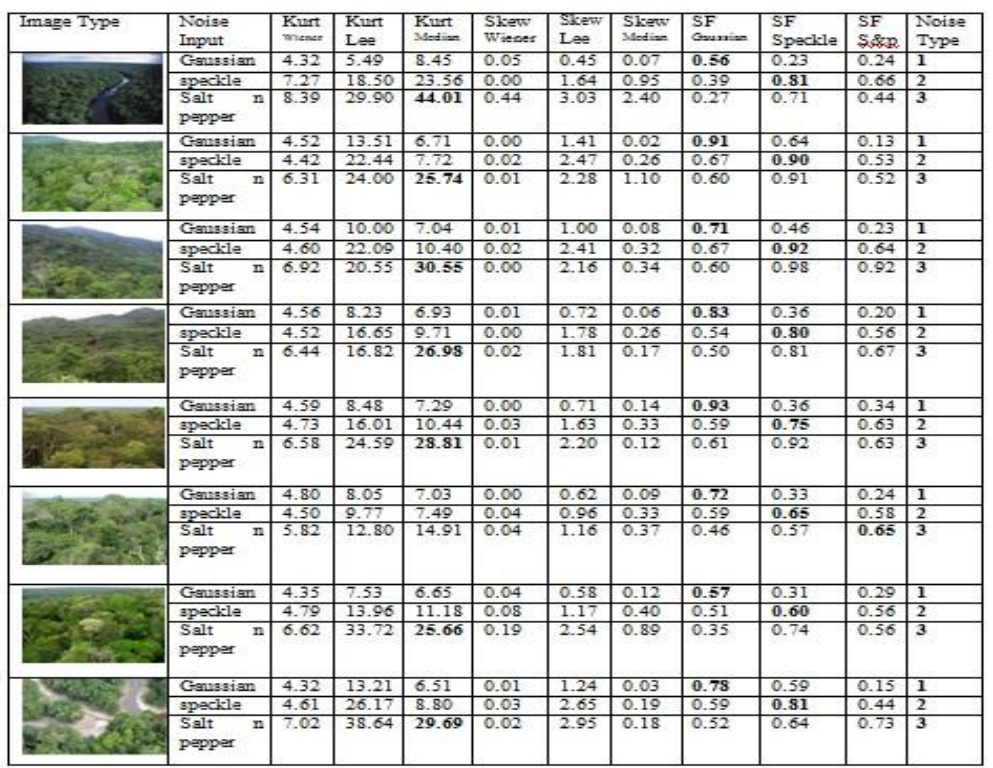

Table 1: Kurtosis, Skewness, and their similarities and also the identified noise types

\section{CONCLUSION}

An automatic noise identification method is proposed in this paper which is globalized for all images. The proposed technique is quite general in nature and can be used with a variety of de-noising filters. Experimental results show that method is appropriate for all types of images. The method is easy to implement and accurate for $\mathrm{M} \mathrm{x} \mathrm{N}$ images. The method is quite general in nature and can be used with a variety of denoising filters. The results of simulation studies seem to indicate that the method is capable of accurately identifying the type of noise.

\section{References}

[1] Yixin Chen, Manohar Das (2007), "An Automated Technique for Image Noise Identification Using a Simple Pattern Classification Approach", IEEE International conference on Circuits and systems, pp.819-822.

[2] Lionel Beaurepaire, Kacem chedi and Benoit Vozel (1997), "Identification of the nature of noise and estimation of its statistical parameters by analysis of local histograms", IEEE International conference on Acoustics, Speech and Signal Processing, Volume: 4, pp. 2805-2808

[3] Dr. P. Subashini, Bharathi.P. T (2011), "Automatic Noise Identification in Images using Statistical Features", International Journal of Computer Science and Technology, Vol. 2, Issue 3, pp. 467-471.

[4] Raina , Shamik Tiwari ,Deepa Kumari ,Deepika Gupta (2012), "An approach for image noise identification using minimum distance classifier", International Journal of Scientific \& Engineering Research Volume 3, Issue 4, pp 1-4

[5] Karibasappa K.G and K. Karibasappa (2010), "Identification and Removal of Impulsive noise using Hypergraph Model”, IJCSE International Journal on Computer Science and Engineering, Vol. 02, No. 08, pp. 2666-2669.

[6] K. Chehdi, M. Sabri(1992), "A New Approach to identify the nature of noise affecting an image, IEEE on Accoustics, Speech, Signal Processing”, Vol.3, pp. 285-288

[7] B. Vozel, K. Chehdi, L. Klaine, Vladimir V. Lukin, Sergey K. Abramov, "Imitation of its statistical parameters by using unsupervised variational classification”, IEEE International Conference on Acoustics, Speech and Signal Processing, Vol. 2, pp. 841-844.

[8] T. Santhanam and S. Radhika (2010), "A Novel Approach to Classify Noises in Images Using Artificial Neural Network”, Journal of Computer Science, Vol 1, Pp. 506-510.

[9] Shamik Tiwari, Ajay Kumar Singh, V.P. Shukla (2011), "Statistical Moments based Noise Classification using Feed Forward Back Propagation Neural Network", Proc. International Journal of Computer Applications, Vol. 18, No. 2, pp. 36-40

[10] K. Chehdi(1993). "Automatic identification of noises for an optimal filtering", Proceedings IEEE CCSP 93, pp. 474-477.

[11] L. Beaurepaire and K. Chehdi (1996). "Identification of degraded image by a multiplicative or additive noise," Proceedings EUSIPCO-96, vol 11: pp. 899-902.

[12] Balasubramaniam Sridhar (2008), "Noise type and statistical parameters identification," Computer Vision Project Report.

[13] A. Rosenfeld (1995), “Digital Image Processing”, Academic Press, San Diego, 1982.Hyung Lee-Kwang, Keon-Myung Lee. Fuzzy hypergraph and fuzzy partition. IEEE Transactions on Systems, Man and Cybernetics, vol.25(1): pp.196-201.

[14] Mohammed Ghouse, Dr. M. Siddappa (2011), "Adaptive Techniques based high impulsive noise detection and reduction of a digital image", Journal of Theoretical and Applied Information Technology 2011 Jatit \& Lls.

[15] V.R.Vijay Kumar, S.Manikandan, P.T.Vanathi, P.Kanagasabapathy and D.Ebenezer (2008), “Adaptive Window Length Recursive Weighted Median Filter for Removing Impulse Noise in Images with Details Preservation", ECTI Transactions on Electrical Eng., Electronics, and Communications Vol.6, No.1.

[16] D.Maheswari, Dr.V.Radha (2010), "Noise Removal in Compound Image Using Median Filter", International Journal on Computer Science and Engineering Vol. 02, No. 04, pp.1359-1362.

[17] Milindkumar V. Sarode, Prashant R. Deshmukh (2011), "Reduction of Speckle Noise and Image Enhancement of Images Using Filtering Technique”, International Journal of Advancements in Technology, Vol 2, No 1.

[18] M. Oussalah and J. De Schutter (2010), “Adaptive Kalman Filter For Noise Identification”, ISMA25, pp 1225-1232 